\title{
Sylvie Thorel-Cailleteau, Splendeurs de la Médiocrité. Une idée du Roman
}

\section{Nicola Ferrari}

\section{(2) OpenEdition}

1 Journals

\section{Edizione digitale}

URL: http://journals.openedition.org/studifrancesi/8423

DOI: 10.4000/studifrancesi.8423

ISSN: 2421-5856

\section{Editore}

Rosenberg \& Sellier

\section{Edizione cartacea}

Data di pubblicazione: 1 mai 2009

Paginazione: 224-225

ISSN: 0039-2944

\section{Notizia bibliografica digitale}

Nicola Ferrari, «Sylvie Thorel-Cailleteau, Splendeurs de la Médiocrité. Une idée du Roman», Studi Francesi [Online], 157 (LIII | I) | 2009, online dal 30 novembre 2015, consultato il 12 janvier 2021. URL: http:// journals.openedition.org/studifrancesi/8423 ; DOI: https://doi.org/10.4000/studifrancesi.8423

Questo documento è stato generato automaticamente il 12 janvier 2021.

\section{(c) $(1) \&$}

Studi Francesi è distribuita con Licenza Creative Commons Attribuzione - Non commerciale - Non opere derivate 4.0 Internazionale. 


\title{
Sylvie Thorel-Cailleteau, Splendeurs de la Médiocrité. Une idée du Roman
}

\author{
Nicola Ferrari
}

\section{NOTIZIA}

SYLVIE THOREL-CAILLETEAU, Splendeurs de la Médiocrité. Une idée du Roman, Genève, Droz, 2008 , pp. 256.

1 Maupassant l'aveva sepolto offrendogli l'onore di un'epigrafe sorniona e irresistibile: il tentativo di discriminare quali opere letterarie siano da considerare romanzi e quali no rappresenterebbe una perspicacia critica che somiglia molto all'incompetenza. Due secoli di scritture e riletture avevano tratteggiato un'immagine d'insieme proteiforme e indeterminabile, dimostrando il fallimento del programma intrapreso nel 1670 da Huet con la sua celebre Lettre sur l'origine des romans: elaborare una teoria del romanzo come genere. Contre Maupassant, l'importante saggio di THOREL-CAILLETEAU, applica alla letteratura dei tre secoli successivi il progetto teorico di Huet, impegnandosi a dimostrarne la plausibilità.

2 È possibile articolare un percorso critico che giunga all'astratta scrittura di Beckett, partendo dalla ricezione seicentesca degli archetipi antichi (Eliodoro: l'architettura formale e il repertorio di temi delle Etiopiche persistenti nella narrativa francese fino al Settecento; Ovidio: la rivendicata priorità di un modello prosastico, antiepico nelle Metamorfosi e, soprattutto, le lettere amorose delle Heroides, palinsesto di tutto il romanzo epistolare fino a Rousseau; Virgilio: il libro quarto dell'Eneide, rivoluzionaria introduzione del tema erotico nell'epica, invocata legittimazione dell'alterità sentimentale della scrittura romanzesca)? Alla ricerca di un filo che permetta di dare un senso al labirinto di forme e soggetti riuniti, dal Seicento all'Ottocento sotto la stessa indicazione di romanzo, molte sono le figure - metafore, temi, luoghi, e personaggi delle quali si possono scoprire e raccontare suggestive permanenze: l'allegoria ovidiana della narrazione come tela (con la sua connotazione di attività femminile - del lavoro, 
della quotidianità, del sentimento - opposta all'epica maschile - della guerra, dell'eccezionalità, dell'azione); il mito di Medusa (che, come la realtà, ci affascina e ci perde); le pustole del vaiolo (che violano e feriscono la Grazia femminile superficiale, scoprendone i baratri di interno orrore o, al contrario, rivelando il noumeno di una bellezza finalmente soprasensibile, da Laclos a Zola via Balzac e Barbey d'Aurevilly); la storia dell'oscuro capo della polizia parigina, Vidocq, trasfigurata in archetipo romanzesco da Hugo, Sue, Dumas e la successiva letteratura popolare; la casa, come spazio polifonico di un universo (enciclopedico) di storie che si sfiorano, intrecciano, sovrappongono, da Balzac a Perec; l'esplorazione dei bassifondi parigini come inferno dantesco e altro mondo 'mohicani' alla Fenimore Cooper (ancora da Hugo a Sue). Ma dietro il riconoscimento di queste costanti parziali, attraverso una ricca esemplificazione - acutamente analizzata per trasformare i romanzi stessi in vere e proprie teorie del romanzo - il filo unificante viene finalmente ritrovato in quella nozione di mediocrità, alla quale si intitola il saggio. Fortemente (ma non dichiaratamente) debitrice rispetto al modello elaborato da Auerbach per la sua storia della rappresentazione narrativa della realtà in Occidente, il romanzo si definisce come genere mediocre. L'originaria nozione classica - insieme metafisica e morale - di intermediazione tra gli estremi, indirizza il destino del romanzo sulle vie, tanto morfologiche che contenutistiche, della secolarizzazione (la sua esplorazione si ripiega sulla finitezza umana, nel mondo sublunare, che non accede ai celesti spazi), dell'ibridazione (per quanto si vogliano distinguere forme serie e idealizzanti del romanzo da quelle comico-realistiche, tragico e comico risultano sempre ingredienti compresenti, nell'impasto romanzesco), della prosa (le strutture metriche, i versi, le rime, seguendo Huet contro Tasso, costituiscono un vettore linguistico del sublime, statutariamente negato al romanzo) e della pura testualità (il partito antiplatonico della rappresentazione come assenza della voce, autonomia della scrittura sul soggetto).

3 Seguendo l'evoluzione delle forme e degli oggetti di rappresentazione, nello slittare delle modalità applicative di questa cornice di genere, la storia del romanzo moderno appare come progressivo esilio del romanzesco, dalla sua iniziale messa in scena alla celebrazione (flaubertiana) del suo rimpianto. In questo passaggio, però, attraversando l'ottocento ctonio e perturbante, cambia radicalmente la definizione semantica dell'asse concettuale, intorno al quale ruota lo statuto del romanzo: la mediocrità come rivendicazione di medietà stilistica, si rivela attrazione per il prosaico, - per l'irresistibile precipitare dei valori etici e delle virtù nella rappresentazione della canaille -, infine rivendicata indifferenza all'oggetto di rappresentazione, riconosciuto privo di qualsiasi tratto intrinsecamente caratterizzante. Grazie a questa mobilità del termine di riferimento concettuale, la sottile trattazione dello sviluppo del genere riesce a risolvere come continuità gli scambi dissestati dei paradigmi di poetica. Appare però, con chiarezza, che il saggio non consegue il progetto proposto di una teoria del romanzo come genere, quanto piuttosto definisca e articoli la genealogia storica del récit beckettiano, individuandone con acume le premesse possibili nella narrazione moderna.

4 Elaborazione, che approfondisce e arricchisce di riflessioni analitiche - senza mutare impianto e istanze programmatiche generali - del saggio La poesia della mediocrità, apparso nel 2006 all'interno del quarto volume, dedicato a motivi luoghi ed eroi, del Romanzo di Franco Moretti, questi Splendeurs de la Médiocrité - condividono con la grande opera(zione) einaudiana un paradossale destino di successo attraverso il 
fallimento. Se, nel caso del progetto enciclopedico, la moltiplicazione degli spazi, dei tempi e delle forme, negava la liceità di una trattazione unitaria di fenomeni solo nominalmente coincidenti (ma proprio l'irrisolta tensione tra l'unicità dell'etichetta $\mathrm{e}$ l'irriducibile alterità delle sostanze scopriva imprevedibili giochi di senso, parentele, derivazioni, deviazioni), specularmente, nel caso di Thorel-Cailleteau, la frustrazione dell'ambizioso programma monistico - l'idea del Romanzo - permette di offrire, nei propri margini e interstizi, un tesoro di osservazioni critiche profondamente innovative, felici intuizioni che il taglio programmatico dona agli autori più consolidati del canone. Infatti: l'indagine sullo statuto di genere determina un metodo di interrogazione dei testi di approccio eminentemente comparatistico, ancorché applicato di fatto alla sola esperienza francese - le sporadiche esemplificazioni forestiere (Poe, il Conrad dell'Agente segreto, il Dostoevskij delle Memorie del sottosuolo e il Kafka della Tana) trovano collocazione solo nell'esplicita assimilazione alla letteratura di riferimento. La libertà delle associazioni e dei confronti, indipendentemente dalle consuete (o consuetudinarie) linee di derivazione storiografiche, insieme alla prospettiva anamorfica che cerca di leggere unitariamente paradigmi storicamente distanti tra loro, come asintotica tensione verso quella nozione - notturna, funeraria, nihilista - di récit, che, derivata da Blanchot, definisce il solitario cupio dissolvi beckettiano, permette di leggere euristicamente il fallimentare tentativo di sottrarsi al monito di Maupassant. Chiudendo il libro, al concetto di mediocrità non dobbiamo la formula risolutiva di un genere indeterminabile e sfuggente, che continua a negarsi a ogni tentativo di codificazione; ma l'affascinante narrazione di tre storie differenti (o forse la stessa, tutta francese, che si può leggere in tre modi): la storia, si è detto, che conduce dalle interiorità segrete, sfuggite, rapite nelle reti di sguardi e sospiri del romanzo di Mme de La Fayette all'interna voce del non romanzo di Beckett perduta in un tempo deserticamente presente, in uno spazio senza dimensione, in attesa (e contemplazione) della propria, procrastinata e inevitabile, dissoluzione; una storia della letteratura francese moderna, attraversata con gesto assai personale e criticamente creativo (dalla scoperta secentesca del sentimento individuale e della intepretazione narrativa dei modelli conversazionali, alle eredità di Goncourt in Zola, di Zola in Proust, di Proust e Céline, associati, in Beckett); infine, una Storia nazionale, che riflette alcuni dei suoi nodi paradigmatici - la reazione giansenista alla politica di Louis XIV o le ferite generazionali ed epocali inferte dal Terrore postrivoluzionario sulla superficie incrinata e frammentaria della scrittura romanzesca. 